\title{
Surgical Treatment of Type 2 Diabetes
}

\author{
Matthias Blüher
}

Editorial to
accompany the
article:
"Renal Function in
Type 2 Diabetes
Following Gastric
Bypass-
A Prospective
Cohort Study in
Mildly Obese
Insulin-dependent
Patients" by
Adrian T. Billeter
et al. in this issue
of Deutsches
Ärzteblatt
International

Department of Internal Medicine, University

Hospital Leipzig:

Prof. Dr. med. Blüher
$\mathbf{W}$ ith a prevalence of $5-8 \%$, type 2 diabetes is one of the most common metabolic disorders in Germany. Chronic hyperglycemia goes hand in hand with an increased risk for microvascular complications, such as diabetic retinopathy, neuropathy, and nephropathy. This makes type 2 diabetes one of the most common causes of loss of eyesight, renal failure, and amputation of the lower limb (1). Diabetic nephropathy is the most common complication of type 2 diabetes and results in a substantially impaired quality of life for those affected. It also incurs high costs to the healthcare system - owing to expensive dialysis therapy, among others (2).

In addition to age, familial predisposition, and certain lifestyle factors, overweight is one of the factors promoting the manifestation of type 2 diabetes. Weight loss can help to delay the manifestation of type 2 diabetes, and weight reducing measures constitute basic treatment (lifestyle management/intervention) in patients with type 2 diabetes. Individually agreed therapeutic goals (normalizing blood glucose concentrations, weight reduction) are, however, rarely achieved by means of basic therapy alone; the guideline conform standard in this setting is stepwise escalation of pharmacotherapy for type 2 diabetes (1).

\section{Metabolic surgery and diabetic nephropathy}

Since weight reduction for patients with type 2 diabetes and obesity is hard to achieve by means of conservative strategies, in recent years, metabolic surgery-especially Roux-en-Y gastric bypass surgery-has become an established therapeutic alternative in patients with obesity-associated type 2 diabetes. New pharmacotherapy options for type 2 diabetes are often not able to prevent disease progression and complications of diabetes. Although the positive effects of metabolic surgery regarding all-cause mortality, weight reduction, blood glucose control, and reduction of the need for medication have been widely documented $(3,4)$ for up to medium term diabetes remission rates $(60-90 \%)$, their effects on diabetes specific complications have not been systematically investigated. The pilot study reported by Billeter et al. therefore focuses on the question of whether metabolic surgery can yield improved parameters of diabetic nephropathy (2).

The authors showed that in 20 patients with type 2 diabetes who did not reach their target measurements in spite of optimized insulin treatment, the parameter of renal function improved notably for up to 24 months after Roux-en-Y gastric bypass surgery (2). Of note is the fact that the patients included in the study reported by Billeter et al. had a mean body mass index (BMI) of $32.8 \mathrm{~kg} / \mathrm{m}^{2}$. Up to now, the indication for metabolic surgical procedures existed in patients with obesity $\left(\mathrm{BMI}>40 \mathrm{~kg} / \mathrm{m}^{2}\right)$ or with a BMI $>35 \mathrm{~kg} / \mathrm{m}^{2}$ and severe comorbidities (for example, type 2 diabetes). Should we rethink our indication criteria for metabolic surgery on the basis of the findings of the study reported by Billeter et al.?

\section{Indication for surgical treatment in type 2 diabetes}

In principle, pharmacotherapy for type 2 diabetes can guarantee a lifetime of safe and effective blood glucose control. Randomized prospective clinical studies $(4,5)$, however, in which surgical (combined with optimized medical) treatment was obviously superior to medication treatment triggered a discussion of whether metabolic surgery may be the better strategy for treating type 2 diabetes, but also whether it may help prevent disease progression and diabetes related complications (6). Studies conducted to date that compared surgical and medical diabetes treatment were (with the exception of the SOS Study [3]) conducted over a relatively short time period) (up to 3 years) and with small numbers of participants.

Another question that remains unanswered is whether the promising results of the randomized clinical studies can be confirmed under routine conditions. In the guideline on the treatment of obesity, surgical measures are included as an established option in the stepwise approach to therapy (7), the national practice guideline for the treatment of type 2 diabetes interestingly does not mention the surgical option (1). This contrasts with the International Diabetes Federation, which declared as early as in 2011 that for persons with obesity-associated type 2 diabetes who are unable to reach their therapeutic goals $(\mathrm{HbAl} c<7.5 \%)$ by employing conservative strategies, metabolic surgery is an appropriate treatment modality for type 2 diabetes (8).

\section{Risks}

In terms of the individual decision to have surgery for type 2 diabetes, risks such as acute perioperative complications and longer term deficiencies in terms of the supply of micronutrients or psychological problems need to be considered.

In routine clinical practice, the number of patients who undergo surgical treatment for type 2 diabetes is low compared to the large number of patients with an indication for bariatric surgery. Patients' fears of the 
procedure and reservations among treating doctors are likely to have an important role in this. In fact, surgical therapy of obesity and type 2 diabetes entails short term (perioperative mortality: $0.1-0.3 \%$, complications related to the procedure: $2-4 \%)(9,10)$ and longer term risks:

- Deficiencies in certain micronutrients,

- dumping,

- osteoporosis,

- skin wrinkles,

- weight gain,

- psychological problems,

- increased suicidality.

\section{Conclusions}

Even in the future, surgical therapy is not likely to become the treatment of choice for most patients with type 2 diabetes. Modern antidiabetic drugs and modified insulin therapies enable safe, long term blood glucose control in the target range while avoiding hypoglycemia and weight gain, while maintaining a good quality of life for those affected.

On the other hand, it should increasingly be the responsibility of diabetes specialists to identify those patients with type 2 diabetes who would experience sustained benefits from early surgical therapy. Before surgical therapy is embarked on, therapeutic attempts using conservative basic measures and medication treatment should be explored exhaustively.

The study reported by Billeter and colleagues provides support for the idea that metabolic surgery should in the future be considered at an earlier point in time for the treatment of type 2 diabetes even in patients with a BMI of $25-35 \mathrm{~kg} / \mathrm{m}^{2}$, and should not be regarded as the last therapeutic option. However, first of all, the promising data from this pilot study will need to be confirmed in long term randomized and controlled studies. Therapeutic success after metabolic surgery largely depends on embedding the procedure in a multimodal therapeutic concept for the optimal preparation and structured, interdisciplinarily organized long term follow-up care for the patient.

\section{Conflict of interest statement}

Prof. Blüher has received consultancy fees from AstraZeneca, Bayer, Boehringer-Ingelheim, Johnson\&Johnson, Lilly, Novo Nordisk, Novartis, Pfizer, and Sanofi.

\section{REFERENCES}

1. Pfeiffer AFH, Klein $\mathrm{HH}$ : The treatment of type 2 diabetes. Dtsch Arztebl Int 2014; 111: 69-82.

2. Billeter AT, Kopf S, Zeier M, et al.: Renal function in type 2 diabetes following gastric bypass - a prospective cohort study in mildly obese insulin-dependent patients. Dtsch Arztebl Int 2016; 113: 827-33.

3. Sjöström L, Narbro K, Sjöström CD, et al.: Effects of bariatric surgery on mortality in Swedish obese subjects. N Engl J Med 2007; 357: 741-52

4. Schauer PR, Bhatt DL, Kirwan JP, et al., STAMPEDE Investigators: Bariatric surgery versus intensive medical therapy for diabetes-3-year outcomes. N Engl J Med 2014; 370: 2002-13.

5. Mingrone G, Panunzi S, De Gaetano A, et al.: Bariatric surgery versus conventional medical therapy for type 2 diabetes. N Engl J Med 2012; 366: 1577-85.

6. Rubino F: Medical research: Time to think differently about diabetes. Nature 2016; 533: 459-61.

7. Wirth A, Wabitsch M, Hauner H: Clinical practice guideline: The prevention and treatment of obesity. Dtsch Arztebl Int 2014; 111: 705-13.

8. Dixon JB, Zimmet P, Alberti KG, Rubino F, International Diabetes Federation Taskforce on Epidemiology and Prevention: Bariatric surgery: an IDF statement for obese type 2 diabetes. Diabet Med 2011; 28: 628-42.

9. Longitudinal Assessment of Bariatric Surgery (LABS) Consortium Flum DR, Belle SH, King WC, et al.: Perioperative safety in the longitudinal assessment of bariatric surgery. N Engl J Med 2009; 361: 445-54.

10. Birkmeyer NJ, Dimick JB, Share D, et al., Michigan Bariatric Surgery Collaborative: Hospital complication rates with bariatric surgery in Michigan. JAMA 2010 304: 435-42.

\section{Corresponding author:}

Prof. Dr. med. Matthias Blüher

Department für Innere Medizin

Universität Leipzig, Liebigstr. 20

bluma@medizin.uni-leipzig.de

\section{Cite this as:}

Blüher M: The role of surgery in type 2 diabetes. Dtsch Arztebl Int 2016; 113 : 825-26. DOI: 10.3238/arztebl.2016.0825 